\title{
On Orthogonal Polynomial Matrices
}

\author{
G. Ramesh ${ }^{1}$, R. Gajalakshmi ${ }^{2}$ \\ ${ }^{I}$ (Department of Mathemtics, Govt Arts College (Auto), Kumbakonam, Tamil Nadu, India) \\ ${ }_{2}^{2}$ (Department of Mathemtics, Periyar Maniammai University, Thanjavur, Tamil Nadu, India)
}

\begin{abstract}
The concept of orthogonal polynomial matrices are introduced. Some properties and characterization for polynomial orthogonal matrices are obtained.

Keywords: Polynomial matrices, orthogonal matrices, symmetric matrices.
\end{abstract}

\section{Introduction}

In matrix theory, we come across some special types of matrices and two among them are symmetric matrix and orthogonal matrix. The term orthogonal matrix was used in 1854 by Charles Hermite in the Cambridge and Dublin Mathematical Journal, although it was not until 1878 that the formal definition of an orthogonal matrix was published by Frobenius. An orthogonal matrix is the real specialization of a unitary matrix and thus always a normal matrix. Orthogonal matrices are important for a number of reasons, both theoretical and practical.

A matrix $A(\lambda)$ is said to be a Polynomial matrix if all entries of $A(\lambda)$ are polynomials. Polynomials and polynomial matrices arise naturally as modeling tools in several areas of applied mathematics, science and engineering, especially in systems theory [2], [4], [5].

In this paper we have introduced polynomial orthogonal matrix and extend some properties of orthogonal matrices to orthogonal polynomial matrices.

\section{Preliminaries}

Definitions $2.1[1]$

A matrix $\mathrm{A}$ is defined to be a rectangular arrangement of $\mathrm{mn}$ numbers arranged in $\mathrm{m}$ rows and $\mathrm{n}$ columns. If $\mathrm{m}=\mathrm{n}$ tha matrix is a square matrix. $\mathrm{A}^{\mathrm{T}}$ is a matrix obtained from $\mathrm{A}$ by interchanging its rows into columns. If $A^{T}=A$ then $\mathrm{A}$ is said to be symmetric. A is said to be involutory if $A^{2}=I$. It is orthogonal if $A A^{T}=A^{T} A=I$. It is evident that orthogonal matrices are symmetric and determinant value of an orthogonal matrix is unity.

Definition 2.2 [2]

A matrix $A(\lambda)$ is said to be a polynomial matrix if all entries of $A(\lambda)$ are polynomials.

Definition 2.3 [3]

A square polynomial matrix $A(\lambda)$ is said to be symmetric if $A(\lambda)=A(\lambda)^{T}$ in other words all the coefficient matrices of $A(\lambda)$ are symmetric.

Theorem 2.4 [1]

We state here some theorems without proof as they appeared earlier.

If $\mathrm{A}$ and $\mathrm{B}$ are two square orthogonal matrices, then
(i) $\mathrm{AB}$ is an orthogonal matrix.
(ii) $\mathrm{A}+\mathrm{B}$ is not an orthogonal matrix.

Theorem 2.5

If $\mathrm{A}$ is orthogonal then $\operatorname{det} A= \pm 1$.

Definition 3. 1

\section{Polynomial Orthogonal Matrix}

A polynomial orthogonal matrix is a polynomial matrix whose coefficient matrices are orthogonal.

Example 3.2

$$
\text { Let } A(\lambda)=\left(\begin{array}{ccc}
\frac{3}{5}-\frac{\lambda}{3}-\frac{7}{9} \lambda^{2} & \frac{4}{5}+\frac{2}{3} \lambda+\frac{4}{9} \lambda^{2} & \frac{2}{3} \lambda+\frac{4}{9} \lambda^{2} \\
\frac{4}{5}+\frac{2}{3} \lambda+\frac{4}{9} \lambda^{2} & -\frac{3}{5}-\frac{\lambda}{3}-\frac{1}{9} \lambda^{2} & \frac{2}{3} \lambda+\frac{8}{9} \lambda^{2} \\
\frac{2}{3} \lambda+\frac{4}{9} \lambda^{2} & \frac{2}{3} \lambda+\frac{8}{9} \lambda^{2} & 1-\frac{\lambda}{3}-\frac{1}{9} \lambda^{2}
\end{array}\right)=A_{0}+A_{1} \lambda+A_{2} \lambda^{2}
$$


Where $A_{0}=\frac{1}{5}\left(\begin{array}{ccc}3 & 4 & 0 \\ 4 & -3 & 0 \\ 0 & 0 & 5\end{array}\right), A_{1}=\frac{1}{3}\left(\begin{array}{ccc}-1 & 2 & 2 \\ 2 & -1 & 2 \\ 2 & 2 & -1\end{array}\right), A_{2}=\frac{1}{9}\left(\begin{array}{ccc}-7 & 4 & 4 \\ 4 & -1 & 8 \\ 4 & 8 & -1\end{array}\right)$

$A_{0} A_{0}{ }^{T}=\frac{1}{5}\left(\begin{array}{ccc}3 & 4 & 0 \\ 4 & -3 & 0 \\ 0 & 0 & 5\end{array}\right) \frac{1}{5}\left(\begin{array}{ccc}3 & 4 & 0 \\ 4 & -3 & 0 \\ 0 & 0 & 5\end{array}\right)=\left(\begin{array}{lll}1 & 0 & 0 \\ 0 & 1 & 0 \\ 0 & 0 & 1\end{array}\right)=I$

$A_{0}{ }^{T} A_{0}=\frac{1}{5}\left(\begin{array}{ccc}3 & 4 & 0 \\ 4 & -3 & 0 \\ 0 & 0 & 5\end{array}\right) \frac{1}{5}\left(\begin{array}{ccc}3 & 4 & 0 \\ 4 & -3 & 0 \\ 0 & 0 & 5\end{array}\right)=\left(\begin{array}{lll}1 & 0 & 0 \\ 0 & 1 & 0 \\ 0 & 0 & 1\end{array}\right)=I$

Hence $A_{0} A_{0}^{T}=A_{0}^{T} A_{0}=I$

$A_{1} A_{1}^{T}=\frac{1}{3}\left(\begin{array}{ccc}-1 & 2 & 2 \\ 2 & -1 & 2 \\ 2 & 2 & -1\end{array}\right) \frac{1}{3}\left(\begin{array}{ccc}-1 & 2 & 2 \\ 2 & -1 & 2 \\ 2 & 2 & -1\end{array}\right)=\left(\begin{array}{lll}1 & 0 & 0 \\ 0 & 1 & 0 \\ 0 & 0 & 1\end{array}\right)=I$
$A_{1}^{T} A_{1}=\frac{1}{3}\left(\begin{array}{ccc}-1 & 2 & 2 \\ 2 & -1 & 2 \\ 2 & 2 & -1\end{array}\right) \frac{1}{3}\left(\begin{array}{ccc}-1 & 2 & 2 \\ 2 & -1 & 2 \\ 2 & 2 & -1\end{array}\right)=\left(\begin{array}{lll}1 & 0 & 0 \\ 0 & 1 & 0 \\ 0 & 0 & 1\end{array}\right)=I$

Hence $A_{1} A_{1}^{T}=A_{1}^{T} A_{1}=I$

$$
\begin{aligned}
& A_{2} A_{2}{ }^{T}=\frac{1}{9}\left(\begin{array}{ccc}
-7 & 4 & 4 \\
4 & -1 & 8 \\
4 & 8 & -1
\end{array}\right) \frac{1}{9}\left(\begin{array}{ccc}
-7 & 4 & 4 \\
4 & -1 & 8 \\
4 & 8 & -1
\end{array}\right)=\left(\begin{array}{lll}
1 & 0 & 0 \\
0 & 1 & 0 \\
0 & 0 & 1
\end{array}\right)=I \\
& A_{2}{ }^{T} A_{2}=\frac{1}{9}\left(\begin{array}{ccc}
-7 & 4 & 4 \\
4 & -1 & 8 \\
4 & 8 & -1
\end{array}\right) \frac{1}{9}\left(\begin{array}{ccc}
-7 & 4 & 4 \\
4 & -1 & 8 \\
4 & 8 & -1
\end{array}\right)=\left(\begin{array}{lll}
1 & 0 & 0 \\
0 & 1 & 0 \\
0 & 0 & 1
\end{array}\right)=I
\end{aligned}
$$

Hence $A_{2} A_{2}{ }^{T}=A_{2}{ }^{T} A_{2}=I$

Theorem 3.3

Proof

A polynomial orthogonal matrix is always symmetric.

Let $A(\lambda)=A_{0}+A_{1} \lambda+A_{2} \lambda^{2}+\ldots \ldots \ldots .+A_{n} \lambda^{n}$ be a polynomial orthogonal matrix. Here the coefficient matrices $A_{i}^{\prime} s$ are orthogonal matrices.

Since orthogonal matrices are symmetric, the coefficient matrices of $A(\lambda)$ are all symmetric.

Hence $A(\lambda)$ is a symmetric matrix.

Example 3.4

Consider the orthogonal polynomial matrix,

$A(\lambda)=\left(\begin{array}{ccc}-1+\lambda^{2} & -\lambda & 0 \\ -\lambda & -1+\lambda^{2} & 0 \\ 0 & 0 & -1-\lambda+\lambda^{2}\end{array}\right)=A_{0}+A_{1} \lambda+A_{2} \lambda^{2}$.

Where $A_{0}=\left(\begin{array}{ccc}-1 & 0 & 0 \\ 0 & -1 & 0 \\ 0 & 0 & -1\end{array}\right), A_{1}=\left(\begin{array}{ccc}0 & -1 & 0 \\ -1 & 0 & 0 \\ 0 & 0 & 1\end{array}\right), A_{2}=\left(\begin{array}{lll}1 & 0 & 0 \\ 0 & 1 & 0 \\ 0 & 0 & 1\end{array}\right)$

Here $A_{0}, A_{1}, A_{2}$ are orthogonal. 
Also $A_{0}^{T}=A_{0}, A_{1}^{T}=A_{1}, A_{2}^{T}=A_{2}$.

That is, $A_{0}, A_{1}, A_{2}$ all are symmetric.

Hence $A(\lambda)$ is symmetric.

Theorem 3.5

If $A(\lambda)$ is a $n \times n$ polynomial orthogonal matrix, then all its coefficient matrices are involutory.

Proof

Let $A(\lambda)=A_{0}+A_{1} \lambda+A_{2} \lambda^{2}+\ldots \ldots \ldots . .+A_{n} \lambda^{n}$ be polynomial orthogonal matrix. Here coefficient matrix $A_{i}{ }^{\prime} s$ are orthogonal matrices.

That is,

Since orthogonal matrices are symmetric

That is, $A_{i}^{T}=A_{i} \quad$ for $i=0,1,2, \cdots, n$.

Hence $A_{i} A_{i}^{T}=A_{i} A_{i}=A_{i}^{2}=I \quad$ for $i=0,1,2, \cdots, n$.

Hence each $A_{i}$ is involutory.

Example 3.6

Let $A(\lambda)=\left(\begin{array}{ccc}\frac{3}{7} \lambda & -1-\frac{2}{7} \lambda & -\frac{6}{7} \lambda \\ -1-\frac{2}{7} \lambda & \frac{6}{7} \lambda & -\frac{3}{7} \lambda \\ -\frac{6}{7} \lambda & -\frac{3}{7} \lambda & -1-\frac{2}{7} \lambda\end{array}\right)=A_{0}+A_{1} \lambda$

Where $A_{0}=\left(\begin{array}{ccc}0 & -1 & 0 \\ -1 & 0 & 0 \\ 0 & 0 & -1\end{array}\right), A_{1}=\frac{1}{7}\left(\begin{array}{ccc}3 & -2 & -6 \\ -2 & 6 & -3 \\ -6 & -3 & -2\end{array}\right)$

$A_{0}^{2}=\left(\begin{array}{ccc}0 & -1 & 0 \\ -1 & 0 & 0 \\ 0 & 0 & -1\end{array}\right)\left(\begin{array}{ccc}0 & -1 & 0 \\ -1 & 0 & 0 \\ 0 & 0 & -1\end{array}\right)=\left(\begin{array}{lll}1 & 0 & 0 \\ 0 & 1 & 0 \\ 0 & 0 & 1\end{array}\right)=I$

Hence $A_{0}^{2}=I$

$A_{1}^{2}=\frac{1}{7}\left(\begin{array}{ccc}3 & -2 & -6 \\ -2 & 6 & -3 \\ -6 & -3 & -2\end{array}\right) \frac{1}{7}\left(\begin{array}{ccc}3 & -2 & -6 \\ -2 & 6 & -3 \\ -6 & -3 & -2\end{array}\right)=\left(\begin{array}{lll}1 & 0 & 0 \\ 0 & 1 & 0 \\ 0 & 0 & 1\end{array}\right)=I$

Hence $A_{1}^{2}=I$

Hence all coefficient matrices $A_{0}, A_{1}$ are involutory.

Theorem 3.7

If $A(\lambda)$ is a polynomial orthogonal matrix if and only if $[A(\lambda)]^{T}$ is polynomial orthogonal matrix.

Proof

Let $A(\lambda)=A_{0}+A_{1} \lambda+A_{2} \lambda^{2}+\ldots \ldots \ldots . .+A_{n} \lambda^{n}$ be polynomial orthogonal matrix. Here coefficient matrix $A_{i}{ }^{\prime} s$ are orthogonal matrices. That is,

$$
\left.\begin{array}{c}
A_{\mathrm{O}} A^{{ }^{T}}=A_{O}{ }^{T} A_{\mathrm{O}}=I \\
A_{1} A_{1}{ }^{T}=A_{1}{ }^{T} A_{1}=I \\
\ldots \ldots \ldots \ldots \ldots \ldots \ldots \ldots \\
A_{n} A_{n}{ }^{T}=A_{n}{ }^{T} A_{n}=I
\end{array}\right\} .
$$


To prove $[A(\lambda)]^{T}$ is polynomial orthogonal matrix.

From 3. 1

$[A(\lambda)]^{T}=A_{0}{ }^{T}+A_{1}{ }^{T} \lambda+A_{2}{ }^{T} \lambda^{2} \ldots \ldots \ldots \ldots \ldots+A_{n}{ }^{T} \lambda^{n}$

We know that coefficient matrices $A_{i} s$ are orthogonal matrices.

Hence $[A(\lambda)]^{T}$ is a polynomial orthogonal matrix.

Similarly we can prove the converse.

Example 3.8

Consider the orthogonal polynomial matrix,

$A(\lambda)=\left(\begin{array}{cc}\frac{3}{5}+\frac{30}{97} \lambda & \frac{4}{5}+\frac{97}{102} \lambda \\ \frac{4}{5}+\frac{97}{102} \lambda & -\frac{3}{5}-\frac{30}{97} \lambda\end{array}\right)=A_{0}+A_{1} \lambda$

$[A(\lambda)]^{T}=\left(\begin{array}{cc}\frac{3}{5}+\frac{30}{97} \lambda & \frac{4}{5}+\frac{97}{102} \lambda \\ \frac{4}{5}+\frac{97}{102} \lambda & -\frac{3}{5}-\frac{30}{97} \lambda\end{array}\right)=A_{0}+A_{1} \lambda$

Here $A(\lambda)=[A(\lambda)]^{T}$

Now $A(\lambda)=\frac{1}{5}\left(\begin{array}{cc}3 & 4 \\ 4 & -3\end{array}\right)+\left(\begin{array}{cc}\frac{30}{97} & \frac{97}{102} \\ \frac{97}{102} & -\frac{30}{97}\end{array}\right) \lambda$

Where $A_{0}=\frac{1}{5}\left(\begin{array}{cc}3 & 4 \\ 4 & -3\end{array}\right), \quad A_{1}=\left(\begin{array}{cc}\frac{30}{97} & \frac{97}{102} \\ \frac{97}{102} & -\frac{30}{97}\end{array}\right)$

$A_{0} A_{0}{ }^{T}=\frac{1}{5}\left(\begin{array}{cc}3 & 4 \\ 4 & -3\end{array}\right) \frac{1}{5}\left(\begin{array}{cc}3 & 4 \\ 4 & -3\end{array}\right)=\left(\begin{array}{ll}1 & 0 \\ 0 & 1\end{array}\right)=I$.

Also $A_{0}=A_{0}{ }^{T}$.

$A_{1} A_{1}^{T}=\left(\begin{array}{cc}\frac{30}{97} & \frac{97}{102} \\ \frac{97}{102} & -\frac{30}{97}\end{array}\right)\left(\begin{array}{cc}\frac{30}{97} & \frac{97}{102} \\ \frac{97}{102} & -\frac{30}{97}\end{array}\right)=\left(\begin{array}{ll}1 & 0 \\ 0 & 1\end{array}\right)=I$

Also $A_{1}=A_{1}^{T}$.

Theorem 3.9

If $A(\lambda)$ is a polynomial orthogonal matrix then determinant of $A(\lambda)$ is a polynomial in $\lambda$.

Proof:

Let $A(\lambda)=A_{0}+A_{1} \lambda+A_{2} \lambda^{2}+\ldots \ldots \ldots . .+A_{n} \lambda^{n}$ be polynomial orthogonal matrix. Here coefficient matrix $A_{i}{ }^{\prime} s$ are orthogonal matrices. From theorem 2.5, we have $\operatorname{det} A_{i}= \pm 1, \forall i=0,1, \cdots, n$. Then all the coefficient matrices have determinant value \pm 1 .

Hence $\operatorname{det}(A(\lambda))=\sum_{i=0}^{n}\left(\operatorname{det} A_{i}\right) \lambda^{i}$

$$
= \pm 1 \pm \lambda \pm \lambda^{2} \cdots \pm \lambda^{n}
$$

It is a polynomial in $\lambda$. 
Example 3.10

(i). Let $A(\lambda)=\left(\begin{array}{cc}\frac{1}{\sqrt{2}}+\lambda & \frac{1}{\sqrt{2}} \\ \frac{1}{\sqrt{2}} & -\frac{1}{\sqrt{2}}+\lambda\end{array}\right)=A_{0}+A_{1} \lambda$ If

$$
\begin{aligned}
\operatorname{det} A(\lambda) & =\frac{1}{\sqrt{2}}\left(\begin{array}{cc}
1+\sqrt{2} \lambda & 1 \\
1 & -1+\sqrt{2} \lambda
\end{array}\right) \\
\operatorname{det} A(\lambda) & =\frac{1}{\sqrt{2}}[(1+\sqrt{2} \lambda)(-1+\sqrt{2} \lambda)-1] \\
\operatorname{det} A(\lambda) & =\frac{1}{\sqrt{2}}\left[-1+\sqrt{2} \lambda-\sqrt{2} \lambda+2 \lambda^{2}-1\right] \\
\operatorname{det} A(\lambda) & =\frac{1}{\sqrt{2}}\left[2 \lambda^{2}-2\right] \\
\operatorname{det} A(\lambda) & =\frac{2}{\sqrt{2}}\left[\lambda^{2}-1\right]=\sqrt{2}\left[\lambda^{2}-1\right]
\end{aligned}
$$

(ii) Let $A(\lambda)=\left(\begin{array}{cc}-1 & 0 \\ 0 & 1\end{array}\right) \lambda^{0}=A_{0} \lambda^{0}$

$\operatorname{det} A(\lambda)=-1 \neq 0$

(i.e) constant

Hence determinant of a polynomial orthogonal matrix is a polynomial.

Remark 3. 11

The product of two polynomial orthogonal matrices need not be orthogonal.

For, Let $A(\lambda)$ and $B(\lambda)$ be two polynomial orthogonal matrices.

Let $\quad A(\lambda)=A_{0}+A_{1} \lambda+A_{2} \lambda^{2}+\ldots \ldots \ldots+A_{n} \lambda^{n} \quad$ and $\quad B(\lambda)=B_{0}+B_{1} \lambda+B_{2} \lambda^{2}+\ldots \ldots \ldots+B_{n} \lambda^{n} \quad$ be polynomial orthogonal matrices.

Where $A_{0}, A_{1}, A_{2}, \ldots \ldots \ldots, A_{n}$ and $B_{0}, B_{1}, B_{2}, \ldots \ldots \ldots, B_{n}$ are orthogonal matrices.

To prove $A(\lambda) B(\lambda)$ orthogonal.

$$
\begin{gathered}
A(\lambda) B(\lambda)=\left(A_{0}+A_{1} \lambda+A_{2} \lambda^{2}+\ldots \ldots \ldots+A_{n} \lambda^{n}\right)\left(B_{0}+B_{1} \lambda+B_{2} \lambda^{2}+\ldots \ldots \ldots+B_{n} \lambda^{n}\right) \\
=A_{0} B_{0}+\left(A_{1} B_{0}+B_{1} A_{0}\right) \lambda+\ldots \ldots \ldots+\left(A_{0} B_{n}+A_{2} B_{n-1}+\ldots \ldots . .+A_{n} B_{0}\right) \lambda^{n}
\end{gathered}
$$

By the theorem (2.4) product of two orthogonal matrices are orthogonal.

So $A_{0} B_{0}$ is orthogonal matrix. But the second term of the above equation is not possible.Because sum of the two orthogonal matrices need not be orthogonal. Hence the product of two polynomial orthogonal matrices need not be orthogonal

\section{Conclusion}

Here we have extended some properties of orthogonal matrices to polynomial orthogonal matrices. All other properties can also be extended in a similar way.

\section{References}

[1]. David W.Lewis, Matrix Theory (World Scientific Publishing Co.Pte.Ltd, 1991)

[2]. I. Gohberg,, P. Lancaster and L. Rodman, Invariant Subspaces of Matrices ith Applications (Wiley, New York, 1986 and SIAM, Philadelphia, 2006).

[3]. G. Ramesh, P.N. Sudha, “On Polynomial Symmetric and Polynomial Skew Symmetric Matrices" IJSRD - International Journal for Scientific Research \& Development| Vol. 3, issue 06, 2015 | ISSN (online): 2321-0613

[4]. A.I.G. Vardulakis, Linear Multivariable Control (JohnWiley, Chichester, UK, 1991).

[5]. W.A. Wolovich, Linear Multivariable Systems (Springer Verlag, 1974). 